\title{
Neuroprotective effects of high-dose human albumin against traumatic spinal cord injury in rats
}

\author{
Yildirim $\mathrm{T}^{1}$, Okutan $\mathrm{O}^{2}$, Akpinar $\mathrm{E}^{2}$, Yilmaz $\mathrm{A}^{1}$, Isik $\mathrm{HS}^{1}$ \\ Ordu University School of Medicine, Neurosurgery, Ordu, Turkey. mdtimur@hotmail.com
}

\begin{abstract}
BACKGROUND AND OBJECTIVES: Human serum albumin (HA) is a unique multifunctional protein with neuroprotective properties. We aimed to delineate the mechanisms of HA-induced neuroprotection, supresses inflammatory response and lipid peroxidation after spinal cord injury (SCI).

METHODS AND STUDY DESIGN: Adult female Wistar rats weighing 210-250 g were used for the study. The rats were randomly and blindly allocated into five groups. The one-way analysis of variance (ANOVA) for parametric data and Shapiro-Wilk test was used for evaluating the normal distribution of the variables. Kruskal-Wallis for nonparametric data was used to compare groups. Electron and light microscopies were used to demonstrate ultrastructural changes in spinal cord.

RESULTS: The HA group was significantly different from all the other groups $(p<0.05)$. Both MPSS and HA treatments decreased the MPO significantly. HA treatment decreased the lipid peroxidation. HA treatment prevented the worsening of clinical results. In the HA treatment group, the ultrastructure was protected significantly. The neuronal bodies and axonal structures were normal except for some limited edematous spaces.

CONCLUSIONS: HA improves early clinical results, protects spinal cord ultrastructure, and decreases MPO and LPO levels after spinal cord contusion injury (Tab. 3, Fig. 3, Ref. 39). Text in PDF www.elis.sk.

KEY WORDS: human albumin, spinal cord injury, rats.
\end{abstract}

\section{Introduction}

A cascade of neurotoxic events in the spinal cord, including energy failure, glutamate release and activation of local inflammatory response, calcium influx, $N$-methyl-D-Aspartate (NMDA) receptors, formation of nitric oxide (NO), and apoptosis have been proposed to be responsible for secondary injury of the spinal cord after primary insult $(1,2)$. Primary insult results in activation of membrane phospholipases and lipases, excitatory amino acid neurotransmitters, and initation of lipid peroxidation (3). Lipid peroxidation and oxygen free radicals have been suggested to be important factors in secondary damage to the spinal cord. Also, neutrophils are believed to play an important role in the pathogenesis of secondary injury $(4,5,6,7,8,9)$. Neutrophils, in addition to their response, as phagocytes, release histolytic enzymes, reactive oxygen species and proinflammatory factors which lead to further tissue necrosis and inflammation $(6,7,8,9)$.

To date, however, no specific pharmaceutical neuroprotectants have been developed, which, if administered after the onset of acute SCI, would succeed in diminishing the extent of tissue

${ }^{1}$ Ordu University School of Medicine, Neurosurgery, Ordu, Turkey, and ${ }^{2}$ KTO Karatay University School of Medicine, Neurosurgery Department, Konya,Turkey

Address for correspondence: T. Yildirim, Ordu University School of Medicine, Neurosurgery Deparment, 52200 Ordu, Turkey.

Phone/Fax: +904522252342 damage and improving functional results. So, much of the attention has been focused on the secondary injury since it appears to be susceptible to pharmacological intervention in recent years.

Human serum albumin (HA) is a unique multifunctional protein with neuroprotective properties. Evidence from in vitro and in vivo experiments indicates a neuroprotective function for HA neural insult including cerebral ischemia $(10,11,12)$, cerebral edema $(10,13)$, aneurismal subarachnoid hemorrhage (10), contusional brain edema (14). Palesch et al demonstrated the safety and feasibility of administering high-dose HA solution to patients with acute ischemic stroke in a multicenter clinical trial (15).

We have no information about neuroprotective effects of HA in acute SCI. Thus, in this study, to delineate the mechanisms of HA-induced neuroprotection, we have raised a question whether HA supresses inflammatory response and lipid peroxidation after SCI. Electron and light microscopies were used to demonstrate ultrastructural changes in normal and damaged spinal cord. The effect of HA was compared with that of methylprednisolone (MPSS).

\section{Material and methods}

\section{Experimental groups}

The rats were randomly and blindly allocated into five groups, 8 rats each.

Group A (Control): Laminectomy was performed, and nontraumatized spinal cord samples were obtained 24-hrs after surgery. 
Group B (Trauma): Animals underwent traumatic interventions after laminectomy. Injured spinal cord samples were removed 24hrs after trauma.

Group C (MPSS): Animals received single doses of methylprednisolone sodium succinate (30 mg/kg) (Mustafa Nevzat, Istanbul, Turkey) intraperitoneally, immediately after trauma.

Group D (Vehicle): Animals received $1 \mathrm{ml}$ of vehicle solution (saline) intraperitoneally, immediately after trauma.

Group E (HA): Animals received $5 \mathrm{mg} / \mathrm{kg}$ body weight of HA intraperitoneally, immediately after injury.

Twenty-four hours after trauma, animals were functionally evaluated and then anesthesia was reintroduced in the trauma and treatment groups. One-centimeter spinal cord samples were obtained for the assessment of biochemical and microscopic examinations. The rats were decapitated during deep anesthesia.

\section{Spinal cord injury}

Adult female Wistar rats weighing 210-250 g were used for the study. All the rats were anesthetized with xylazine, $10 \mathrm{mg} / \mathrm{kg}$ (Bayer, Istanbul, Turkey) and ketamine hydrochloride, $60 \mathrm{mg} /$ kg (Parke Davis, Istanbul, Turkey). Through a dorsal incision, T7-9 laminectomies were performed in prone position. The SCI was induced with a $40-\mathrm{g} / \mathrm{cm}$ impact (16). After the surgical and traumatic interventions, surgical wounds were closed in layers with silk sutures. Twenty-four hours after trauma, anesthesia was re-introduced. One-cm spinal cord samples were obtained from operated spinal cord area and the animals were killed. The systolic arterial tension, heart rate, and body temperature was continuously monitored during surgical interventions. The protocols used in this study were approved by the local institutional animal care committee.

\section{Determination of spinal-cord-associated myeloperoxidase activ- ity}

MPO activity associated with spinal cord was determinated as described previously $(17,18)$. The MPO activity levels were measured with the method described by Hamada and Taoka (19, 20). Absorbance at $460 \mathrm{~nm}$ after 5 min was measured in a spectrophotometer, and MPO activity was calculated using a standard curve prepared with purified MPO. Results are expressed as units (U) per gram of tissue.

\section{Determination of spinal-cord-associated lipid peroxidation}

The lipid peroxide levels were measured as described previously (21). Absorbance at $532 \mathrm{~nm}$ after 5 min was measured in a spectrophotometer and malonyaldehyde (MDA) was calculated using a standard curve prepared with purified MDA. Tissue lipid peroxidation levels were expressed as nanomol per gram of tissue.

Light microscopic examination: Samples were evaluated according to presence of hemorrhage, cellular edema, necrosis and neutrophil infiltration by Nikon Optiphod (Japan) light microscope. The investigator evaluating the sections was blinded to the group information.

Transmission electron microscopic examination: Samples were evaluated according to a grading system for quantitative
Tab. 1. Grading System for Quantitative Evaluation of Ultrastructural Findings.

\begin{tabular}{lc}
\hline & Grade \\
\hline A. Mitochondria in neurons & 0 \\
Normal & 1 \\
Prominent cristae & 2 \\
Swollen mitochondria & 3 \\
$\quad$ Amorph material accumulation & 0 \\
\hline B. Small-sized myelinated axons & 1 \\
$\quad$ Normal & 2 \\
Separation in myelin configuration & 3 \\
Interruption in myelin configuration & \\
Honeycomb appearance & 0 \\
\hline C. Medium-sized myelinated axons & 1 \\
Normal & 2 \\
Separation in myelin configuration & 3 \\
Interruption in myelin configuration & \\
Honeycomb appearance & 0 \\
\hline D. Large-sized myelinated axons & 1 \\
Normal & 2 \\
Separation in myelin configuration & 3 \\
Interruption in myelin configuration &
\end{tabular}

evaluation which was established based on similar principles of the methods used for evaluating different tissue samples by Jeol JEM 1200 EX trasmission electron microscope (Tokyo, Japon) and photographed (Tab. 1).

\section{Functional evaluation}

The inclined plane is a behavioural task, which assesses the animal's ability to maintain its position for at least $5 \mathrm{~s}$ constitutes the IP score. Behavioural evaluation of the rats was made 24 hours after trauma. Hind limb functions were assessed by the inclined plane technique of Rivlin and Tator (22), and Basso, Beattie, and Bresnahan (BBB) scoring system as described by Basso et al (23). The average of BBB scores from both hind limbs was evaluated.

\section{Statistical analysis}

Firstly, the Shapiro-Wilk test was used for evaluating the normal distribution of the variables (BBBS, IP degrees, LPO, MPO, mito, sample size). The test showed normal distribution of the variables (BBBS, IP degrees, LPO, MPO) ( $>$ > 0.05). Also, the test showed non-normal distribution of the variables (mito, sample size). Secondly, one-way ANOVA was performed to test whether there were any differences among the groups (Control, T, V, HA and MPSS) for BBBS, IP degrees, LPO, MPO. Then, means were separated by using Tukey HSD multiple comparisons. According the test results, BBBS, IP degrees, LPO, MPO values for Control, T, V, HA and MPSS were expressed as n (sample size), mean with standard deviation, minimum and maximum. Finally, Kruskal-Wallis $\mathrm{H}$ test was performed to test whether there were any differences among the groups (Control, T, V, HA and MPSS) for mito and sample size. According the test results, mito and sample size values for Control, T, V, HA and MPSS were expressed as n (sample size), median with IQR, minimum and maximum. Sig- 
86-91

Tab. 2. Statistical findings including MPO, lipid peroxidation, BBB scores, IP degrees.

\begin{tabular}{|c|c|c|c|c|c|c|c|c|c|}
\hline \multirow{2}{*}{ Groups } & \multirow{2}{*}{$\mathrm{n}$} & \multicolumn{2}{|c|}{ Myeloperoxidase activity } & \multicolumn{2}{|c|}{ Lipid peroxidation } & \multicolumn{2}{|c|}{$\mathrm{BBB}$} & \multicolumn{2}{|c|}{ IP Degrees } \\
\hline & & Mean & $\mathrm{SD}$ & Mean & $\mathrm{SD}$ & Mean & SD & Mean & $\mathrm{SD}$ \\
\hline Control & 8 & $2,332 \mathrm{c}$ & 0,002 & $284,0 \mathrm{~d}$ & 0,01 & $21,00 \mathrm{a}$ & 0,000 & 82,63 a & 1,06 \\
\hline $\mathrm{T}$ & 8 & 3,483 a & 0,002 & 515,5 a & 0,01 & $1,38 \mathrm{~d}$ & 0,744 & $38,63 \mathrm{e}$ & 1,06 \\
\hline $\mathrm{V}$ & 8 & $3,474 \mathrm{~b}$ & 0,003 & $515,4 \mathrm{a}$ & 0,00 & $1,38 \mathrm{~d}$ & 0,744 & $41,25 \mathrm{~d}$ & 1,04 \\
\hline HA & 8 & $2,084 \mathrm{~d}$ & 0,003 & $405,2 \mathrm{~b}$ & 0,00 & $3,38 \mathrm{c}$ & 0,744 & $75,75 \mathrm{~b}$ & 1,04 \\
\hline MPSS & 8 & $2,071 \mathrm{e}$ & 0,002 & 400,3 c & 0,01 & $6,25 \mathrm{~b}$ & 1,035 & $72,75 \mathrm{c}$ & 1,04 \\
\hline Total & 40 & 2,689 & 0,660 & 424,1 & 87,41 & 6,68 & 7,509 & 62,20 & 18,74 \\
\hline P-values & & \multicolumn{2}{|c|}{$<0.001$} & \multicolumn{2}{|c|}{$<0.001$} & \multicolumn{2}{|c|}{$<0.001$} & \multicolumn{2}{|c|}{$<0.001$} \\
\hline
\end{tabular}

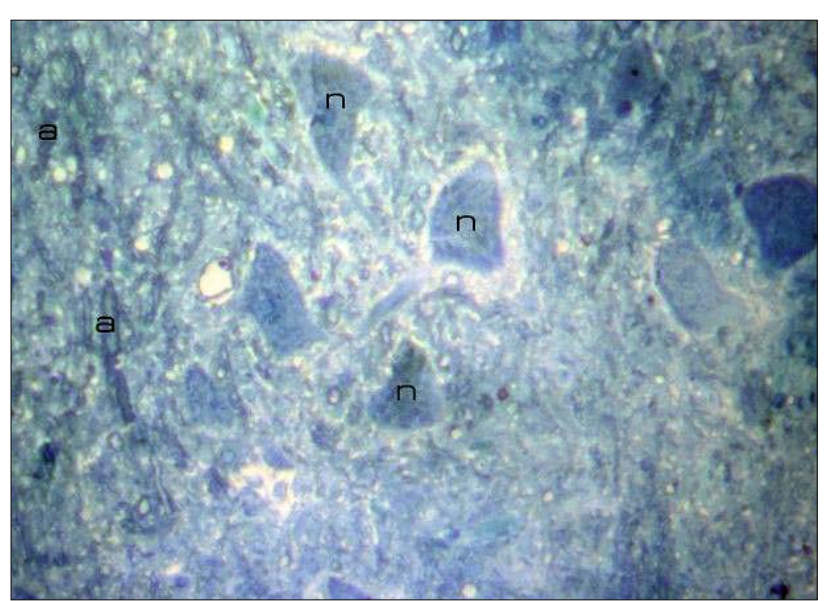

Fig. 1. Light microscopic findings for HA group showing normal neuronal bodies and axonal structures. a: axonal structures, n: neuronal bodies.

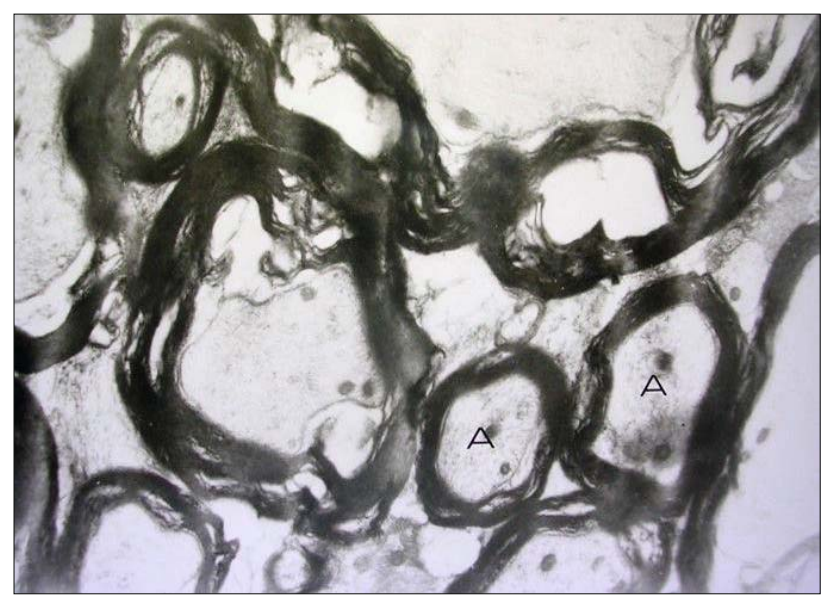

Fig. 2. Electronmicroscopic findings for HA group showing no intraneuronal vacuoles. A: ultrastructure of axons.

nificance was evaluated at $\mathrm{p}<0.05$ for all tests. All computational work was performed by means of SPSS $17.0 \mathrm{~V}$ statistical software e (SPSS 17.0 V; SPSS Inc., Chicago, IL).

\section{Results}

\section{Biochemical findings}

Myeloperoxidase activity: There was a statistically significant difference in the means of MPO activity among control and trauma

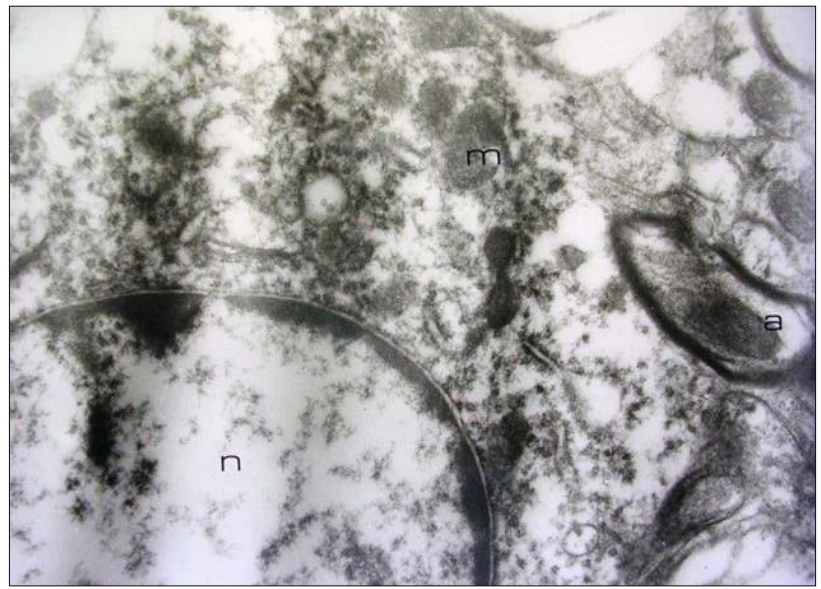

Fig. 3. Electronmicroscopic findings for injury group showing edematous spaces and destroyed myelin sheaths of the axons. a: axon, m:myelin sheat, $n$ : neuronal body.

group ( $\mathrm{p}<0.05$ ). There were statistically significant differences between trauma and both MPSS and treatment groups ( $\mathrm{p}<0.05)$. Both MPSS and HA treatments decreased thr MPO activity significantly (Tab. 2).

Lipid peroxidation levels: There was a statistically significant difference between control and trauma groups in the mean of lipid peroxidation ( $p<0.005)$. LPO levels were measured highest in both trauma and vehicle groups. There was a statistically significant difference between trauma and HA groups $(\mathrm{p}<$ 0.005). HA treatment decreased lipid peroxidation. The difference between HA and MPSS groups was statistically significant ( $\mathrm{p}<$ 0.01) (Tab. 2).

\section{Functional findings}

\section{BBB scores}

The trauma group was not significantly different from the MPSS and vehicle groups ( $p>0.05$ ). The HA group was significantly different from all other groups $(\mathrm{p}<0.05)$. HA treatment prevented the worsening of clinical results (Tab. 2).

Inclined plane degrees

There are statistically significant differences in the means of IP among groups $(\mathrm{p}<0.001)$. Trauma showed a statistically significant decrease in IP values compared with the control group (p $<0.005$ ). Both MPSS and HA groups were significantly different from the trauma group $(\mathrm{p}<0.01)$. Although the differences between MPSS and HA groups were not statistically significant, 
Tab. 3. The mean electron microscopic grading scores of the groups.

\begin{tabular}{lccc}
\hline \multirow{2}{*}{ Factor } & \multicolumn{3}{c}{ Rat Groups } \\
\cline { 2 - 4 } & Trauma & $\begin{array}{c}\text { Methylprednisolon } \\
\text { sodium succinate }\end{array}$ & $\begin{array}{c}\text { Human } \\
\text { Albumin }\end{array}$ \\
\hline Mitochondria & $1.56 \pm 0.52$ & $1.48 \pm 0.53$ & $1.20 \pm 0.51$ \\
\hline $\begin{array}{l}\text { Small sized } \\
\text { myelinated axons }\end{array}$ & $0.63 \pm 0.60$ & $0.57 \pm 0.57$ & $0.47 \pm 0.5$ \\
\hline $\begin{array}{l}\text { Medium sized } \\
\text { myelinated axons }\end{array}$ & $1.06 \pm 0.35$ & $1.03 \pm 0.34$ & $0.97 \pm 0.32$ \\
\hline $\begin{array}{l}\text { Large sized } \\
\text { myelinated axons }\end{array}$ & $1.12 \pm 0.32$ & $1.10 \pm 0.31$ & $1.00 \pm 0.23$
\end{tabular}

HA was not statistically different from control group $(\mathrm{p}>0.05)$ (Tab. 2).

\section{Light microscopic findings}

In the MPSS group, evidence of hemorrhage, cellular edema, and neutrophil infiltration was prominent. In the HA treatment group, the ultrastructure was very well protected. The neuronal bodies and axonal structures were normal except for some limited edematous spaces. Treatment with MPSS also showed obvious ultrastructural protection. However, HA group showed better results than MPSS (Fig. 1).

Transmission electronmicroscopic findings

Ultrastructural findings were evaluated according to a grading system for quantitative evaluation (Tab. 1). Nucleus was found to be normal in all groups. The presence of intraneuronal vacuoles evaluated separately and in trauma, vehicle, and MPSS groups, intraneuronal small- to medium-sized vacuoles were abundant. In the HA group, no intraneuronal vacuoles were observed (Fig. 2). The results of electron microscopic scoring are demonstrated in Table 3.

The injury group showed severe injury with extensive edematous spaces. The myelin sheaths of the axons were destroyed (Fig. 3). In the HA treatment group, the ultrastructure was very well protected. The neuronal bodies and axonal structures were normal except for some limited edematous spaces. Treatment with MPSS also showed obvious ultrastructural protection. However, HA group showed better results than MPSS.

\section{Discussion}

Traumatic injury of the spinal cord causes damage to some axons by physical deformation of spinal cord called primary mechanism $(4,5)$. Mechanical trauma to the spinal cord results in tissue necrosis and initiates a cascade of interactive biochemical and metabolic changes that cause a subsequent progressive injury leading to tissue destruction described as secondary injury. Free radicalinduced lipid peroxidation is believed to play an important role in the pathogenesis of this secondary injury $(4,5,24,25,26)$. But, as lipid peroxidation was demonstrated experimentally to reach peak values immediately after SCI, they appear to be unsuitable targets for pharmacological treatment of SCI in clinical practice (27). Thus, there is a clear and urgent need for a neuroprotective agent with proven efficacy and minimal risk of side effects and toxicity. In our study, LPO levels were measured highest in both trauma and vehicle groups. There was a statistically significant difference between trauma and HA groups $(\mathrm{p}<0.005)$. HA treatment decreased lipid peroxidation.

Albumin has multiple physiologic properties that could be beneficial in central nervous system injury. Recent studies have shown the importance of the neuroprotective effects of HA (11, 28). In the present study, we demonstrated that the intraperitoneal administration of a single dose of HA inhibits lipid peroxidation. Human serum albumin binds avidly to free fatty acids and is thought to play an important physiological role as a scavenger of oxygen free radicals $(12,29,30)$. As albumin is present in relatively high concentrations in both plasma and interstitial fluid, it is strategically situated to scavenge oxygen radicals, to bind to free fatty acids and metal ions, and to interrupt the damaging oxidative process of lipid peroxidation (12). Emerson et al also suggested that albumin inhibits copper ion-dependent lipid peroxidation and formation of highly reactive hydroxyl radical species. (31). Also, albumin provides essential fatty acids like DHA, which can be a very important factor in providing neuronal membrane repair (31). In the present study, nucleus was found to be normal in all groups. Although intraneuronal small- to medium-sized vacuoles were abundant in trauma, vehicle, and MPSS groups, in the HA group no intraneuronal vacuoles were observed (Tab. 3). These results indicate the neuroprotective effects of HA.

Persistent hypoalbuminemia, complete spinal cord injury (ASIA A), and C4 and above neurologic injuries were significantly associated with death. Hypoalbuminemia seems to be an independent predictor of outcome in patients with servical spinal cord injury (32). Hypoalbuminemia is associated with increased morbidity and mortality in various clinical settings and several major diseases (33). In a retrospective study after elective spine surgery, preoperative hypoalbuminemia was found as an independent risk factor for postoperative complications, degenerative and deformity causes (34).

Suziki et al made a study on rats to clarify the fate of extravasated serum protein and tissue reaction in mature and immature brain after blood brain-barrier breakdown. The results suggested that rapid spreading and clearance of extravasted serum proteins may be deeply involved in the characteristic wound healing pattern (35).

Inflammatory responses are important components contributing to secondary injury after SCI. Neutrophils are believed to play a major role in the pathogenesis of secondary injury. Neutrophils appear in the walls of vessels within few hours of injury and large number of neutrophils are observed in the injured tissue from 8 to $24 \mathrm{~h}$ after experimental SCI $(36,37)$. Numerous neutrophils appear in the injured cord 1 day after injury $(36,37)$. The inhibitory effect of HA to the neutrophil infiltration was found to be statistically different from the control group (Table 2). HA can have beneficial effects at sites of inflammation, which are representative of the damages at the side of traumatic spinal cord. We found that parenteral administration of HA has neuroprotective effects on traumatic spinal cord. Our results as well as those of previous studies are in correlation. 


\section{Conclusion}

The results of this study indicate that HA may prevent neuronal apoptosis and conserve neuronal membranes from traumatic spinal injury. HA treatment improves early clinical results, protects spinal cord ultrastructure, and decreases MPO activity and LPO levels after spinal cord contusion injury in rats. Neuroprotective effects of HA have not been studied in traumatic or ischemic SCI models so far. Additional research is also necessary to further evaluate the potential benefits of HA treatment in the management of acute SCI injury in humans. Early corrective action for hypoalbuminemia may help to reduce mortality in patients with CSCI, it has habitually been included among the parameters used for nutritional assessment, and should be used more frequently as a prognostic tool to detect malnutrition and risk of adverse surgical outcomes.

\section{References}

1. Dumont RJ, Okonkwo DO, Verma S et al. Acute spinal cord injury, part I: Pathophysiologic mechanisms. Clin Neuropharmacol 2001; 24 : 254-264.

2. Pan JZ, Ni L, Sodhi A et al. Cytokine activity contributes to induction of inflammatory cytokine mRNAs in spinal cord following contusion. J Neurosci Res 2002; 68: 315-322.

3. Anderson DK, Hall ED. Pathophysiology of spinal cord trauma. Ann Emerg Med 1993; 22: 987-992.

4. Tator CH, Fehlings MG. Review of the secondary injury theory of acute spinal cord trauma with emphasis on vascular mechanisms. J Neurosurg 1991; 75: 15-26.

5. Hauben E, Schwartz M. Therapeutic vaccination for spinal cord injury: helping the body to cure itself. Trends Pharmacol Sci 2003; 24: 7-12.

6. Profyris C, Cheeema SS, Zang DW et al. Degenerative end regenerative mechanisms governing spinal cord injury. Neurobiol Dis 2004; 15: 415-436.

7. Carlson SL, Parrish ME, Springer JE et al. Acute inflammatory response in spinal cord following impact injury. Exp Neurol 1998; 151: 77-88.

8. Yong VW, Chabot S, Stuve $\mathbf{O}$ et al. Interferon beta in the treatment of multiple sclerosis: mechanisms of action. Neurology 1998; 51: 682-689.

9. Liu Z, Pelfrey CM, Cotleur A et al. Immunomodulatory effects of interferon beta-1b in multiple sclerosis. J Neuroimmunol 2001; 112: 153-162.

10. Belayev L, Liu Y, Zhao W, Busto R, Ginsberg MD. Human albumin therapy of acute ischemic stroke: marked neuroprotective efficacy at moderate doses and with a broad therapeutic window. Stroke 2001; 32: 553-560.

11. Belayev L, Pinard E, Nallet H, Seylaz J, Liu Y, Riyamongkol P, Zhao W, Busto R, Ginsberg MD Albumin therapy of transient focal cerebral ischemia: in vivo analysis of dynamic microvascular responses. Stroke 2002; 33: 1077-1084.

12. Belayev L, Saul I, Huh PW, Finotti N, Zhao W, Busto R, Ginsberg MD. Neuroprotective effect of high-dose albumin therapy against global ischemic brain injury in rats. Brain Res 1999; 845: 107-111.
13. Buemi M, Grasso G, Corica F et al. In vivo evidence that erythropoietin has a neuroprotective effect during subarachnoid hemorrhage. Eur J Pharmacol 2000; 392: 31-34.

14. Tomita H, Ito U, Tone O, Masaoka H, Tominaga B. High colloid oncotic therapy for contusional brain edema. Acta Neurochir Suppl (Wien) 1994; 60: 547-549.

15. Palesch YY, Hill MD, Ryckborst KJ, Tamariz D, Ginsberg MD. The ALIAS Pilot Trial: a dose-escalation and safety study of albumin therapy for acute ischemicstroke. II: neurologic outcome and efficacy analysis. Stroke 2006; 37 (8): 2107-2014.

16. Allen AR. Surgery of experimental lesion of spinal cord equivalent to crush injury of fracture dislocation of spinal column. JAMA 1911; 256: 878-880.

17. Gok B, Okutan O, Beskonakli E, Palaoglu S, Erdamar H, Sargon MF. Effect of immunomodulation with human interferon beta on early functional recovery from experimental spinal cord injury. Spine (Phila Pa 1976) 2007; 32 (8): 873-880.

18. Gok B, Solaroglu I, Okutan O, Cimen B, Kaptanoglu E, Palaoglu S. Metoprolol treatment decreases tissue myeloperoxidase activity after spinal cord injury in rats. J Clin Neurosci 2007; 14 (2): 138-142.

19. Taoka Y, Okajima K, Uchiba M et al. Role of neutrophils in spinal cord injury in rats. Neuroscience 1997; 79: 1177-1182.

20. Hamada Y, Ikata T, Katoh S et al. Involvement of an intracellular adhesion molecule-dependent pathway in the pathogenesis of secondary changes after spinal cord injury in rats. J Neurochem 1996; 32: 192-194.

21. Kaptanoglu E, Solaroglu I, Okutan O et al. Erythropoietin exerts neuroprotection after acute spinal cor injury in rats: effects on lipid peroxidation and early ultrastructural findings. Neurosurg Rev 2004; 27 : 113-120.

22. Kaptanoglu E, Caner H, Solaroglu I, Kilinc K. Mexiletine treatmentinduced inhibition of caspase- 3 activation and improvement of behavioral recovery after spinal cord injury. J Neurosurg Spine 2005; 1: 53-56.

23. Basso DM, Beattie MS and Bresnahan JC. A sensitive and reliable locomotor rating scale for open filed testing in rats. Neurotrauma 1995; 12: $1-21$.

24. Schnell L, Fearn S, Klassen $\mathbf{H}$ et al. Acute inflammatory responses to mechanical lesions in the CNS: differences between brain and spinal cord. Eur J Neurosci 1999; 11: 3648-3658.

25. Kallmann BA, Hummel V, Lindenlaub $\mathrm{T}$ et al. Cytokine-induced modulation of cellular adhesion to human cerebral endothelial cells is mediated by soluble vascular cell adhesion molecule-1. Brain 2000; 123 : 687-967.

26. Goussev S, Hsu JY, Lin Y et al. Differential temporal expression of matrix metalloproteinases after spinal cord injury: relation to revascularization and wound healing. J Neurosurg 2002; 99: 188-192.

27. Limone P, Ferrero B, Calvelli P et al. Hypothalamic-pituitary-adrenal axis and cytokine production in MS with or without interferon-beta treatment. Acta Neurol Scand 2002; 105:3 72-377.

28. Ginsberg MD, Hill MD, Palesch YY, Ryckborst KJ, Tamariz D. The ALIAS Pilot Trial: a dose-escalation and safety study of albumin therapy for acute ischemic stroke I: Physiological responses and safety results. Stroke 2006; 37 (8): 2100-2106.

29. Halliwell B. Albumin - an important extracellular antioxidant? Biochem Pharmacol 1988; 37: 569-571. 
30. Halliwell B, Gutteridge JM. The antioxidants of human extracellular fluids. Arch Biochem Biophys 1990; 280: 1-8.

31. Emerson TE. Unique features of albumin: a brief review. Crit Care Med 1989; 17: 690-694.

32. Jin GX, Li L, Cui SQ, Duan JZ, Wang H. Persistent hypoalbuminemia is a predictor of outcome in cervical spinal cord injury. Spine $\mathrm{J}$ 2014; 14 (9): 1902-1908.

33. Bhuiyan MU. Preoperative Assessment of Serum Albumin Level as Risk Factor for Morbidity Following Routine Oncological Surgery. Mymensingh Med J 2016; 25 (2): 277-283.

34. Adogwa O, Martin JR, Huang K et al. Preoperative serum albumin level as a predictor of postoperative complication after spine fusion. Spine (Phila Pa 1976) 2014; 39 (18): 1513-1519.

35. Suzuki M, Motohashi O, Nishino A, Umezawa K, Shida N, Yoshimoto T. Differences in wound healing pattern between mature and immature brain behavior of extravasated serum protein. Acta Neurochir Suppl (Wien) 1994; 60: 434-436.
36. Means ED, Anderson DK. Neurophagia by leucocytes in experimental spinal cord injury. J Neuropatholog Exp Neurol 1983; 42: 707-719.

37. Yang L, Blumbergs PC, Jones NR et al. Early expression and cellular localization of proinflammatory cytokines interleukin-1b, interleukin-6, and tumor necrosis factor-a in human traumatic spinal cord injury. Spine 2004; 29: 966-971.

38. Bohl DD, Shen MR, Mayo BC, Massel DH, Long WW, Modi KD, Basques BA, Singh K. Malnutrition Predicts Infectious and wound Complications following Posterior Lumbar Spinal Fusion, Spine (Phila Pa 1976) 2016; 41 (21): 1693-1699.

39. Chen X, Liu Z, Sun T, Ren J, Wang X. Relationship between nutritional status and mortality during the first 2 weeks following treatment for cervical spinal cord injury. J Spinal Cord Med 2014; 37 (1): 72-78.

Received September 22, 2017. Accepted November 3, 2017. 